\title{
Búsqueda de tendencias en la precipitación anual del estado de Zacatecas, México; en 30 registros con más de 50 años
}

\author{
Search of Trends in Annual Rainfall of the State of Zacatecas, \\ Mexico, in 30 Records over 50 Years
}

\author{
Campos-Aranda Daniel Francisco \\ Profesor Jubilado de la Universidad Autónoma de San Luis Potosí \\ Correo: campos_aranda@hotmail.com
}

Información del artículo: recibido: enero de 2014, reevaluado: abril de 2014, aceptado: junio de 2014

\section{Resumen}

Las primeras predicciones del cambio climático para México han establecido que en general la precipitación disminuirá y la temperatura ambiente aumentará. Por ello, en este trabajo se aborda el estudio de la precipitación anual del estado de Zacatecas, México, para buscar tendencias climáticas, a través de dos enfoques: el primero de carácter estadístico para detectar tendencia o cambios en la media, lo que significa pérdida de homogeneidad en los registros analizados; el segundo de tipo cuantitativo consistente en evaluar el cociente entre la precipitación media reciente y la de largo plazo para observar si se aparta de la unidad. Para estimar una precipitación media estable, únicamente se procesaron 30 estaciones pluviométricas con registros de más de 50 años. Se encontró que no existe evidencia estadística general de tendencia, pues únicamente dos registros mostraron un comportamiento decreciente; sin embargo, la lluvia anual se está reduciendo como lo detecta el cociente $\left(\mathrm{CO}_{15}\right)$ entre la lluvia media de los últimos 15 años y la de largo plazo, cuya moda de 27 valores es 0.975 .

\footnotetext{
Abstract

For Mexico, it has been predicted that climatic change will generally decrease precipitation and increase the mean temperature. In this paper the annual rainfall of the state of Zacatecas, Mexico was studied. Two approaches were used to look for climatic trends: the first one, a statistical approach to detect trends and/or changes in the mean, which means a loss of homogeneity in the records analyzed; the second one, a quantitative approach which consists in evaluating the ratio of the recent average precipitation to the long run, to observe if it deviates from the unit. To estimate a stable average precipitation only 30 rain gauge stations with records of more than 50 years were processed. It was found that there is no general statistical evidence of trend, because only two records showed a decreasing behavior; however, through the ratio $\left(\mathrm{CO}_{15}\right)$ between the average rainfall of the last 15 years and the long run (whose mode of 27 values is 0.975 ) shows a decrease in the annual rainfall.
}

\section{Descriptores:}

- homogeneidad

- persistencia

- tendencia

- cambio en la media

- estacionalidad

- pruebas paramétricas

- pruebas no paramétricas

\section{Keywords:}

- homogeneity

- persistence

- trend

- change in mean

- seasonality

- parametric tests

- non parametric tests 


\section{Introducción}

Durante las dos últimas décadas se han planteado dos preguntas fundamentales en relación con el cambio climático (CC), la primera es si realmente existe a nivel global y a corto plazo; la segunda es si son las actividades humanas las que lo han originado. En 2007, con el $4^{\circ}$ reporte del Panel Intergubernamental sobre Cambio Climático (IPCC), estas preguntas fueron contestadas afirmativa y categóricamente. La consecuencia fundamental del CC es una elevación significativa de la temperatura ambiente del planeta, la cual pone en marcha una serie de efectos como el derretimiento de los glaciares y polos, con la consecuente elevación del nivel del mar, el incremento en la evaporación en suelos y superficies de agua, lo cual origina un cambio en los patrones de lluvia, generándose una alteración de los ecosistemas, etcétera (Raynal, 2011).

Los últimos escenarios del IPCC establecen, en términos generales, que en México, por su ubicación geográfica, las precipitaciones disminuirán y las temperaturas aumentarán (Martínez y Aguilar, 2008). Además, debido a sus condiciones socioeconómicas, México presentará una elevada vulnerabilidad al CC. Las afectaciones al ciclo hidrológico de las cuencas, establecen la necesidad de revisar con detalle los planes actuales y futuros sobre el aprovechamiento de los recursos hídricos (Martínez y Patiño, 2010). Entonces, para formular las medidas de adaptación que permitan mitigar los efectos del CC, con el propósito de alcanzar niveles razonables de sustentabilidad en el uso del agua, primero se deben identificar los cambios en tales variables meteorológicas, tanto en forma cualitativa como cuantitativa (Kundzewicz y Robson, 2004; Madsen et al., 2013).

El objetivo de este trabajo consiste en buscar cambios significativos en la precipitación anual del estado de Zacatecas, México. Lo anterior se hizo mediante dos enfoques, el primero de tipo estadístico intenta detectar componentes determinísticas como tendencia y cambios en la media en los registros de lluvia y el segundo de carácter cuantitativo, evalúa un cociente entre la precipitación media reciente y la de largo plazo del registro analizado. Para ello, se procesaron 30 registros de precipitación anual con más de 50 años y se utilizaron tres pruebas estadísticas generales y 13 específicas, citando sus referencias básicas y describiendo con detalle el procedimiento operativo de las relativas a la tendencia en los apéndices. Los resultados indican que en el estado de Zacatecas no existe evidencia estadística general del CC, pues los registros no muestran tendencia, sin embargo, la lluvia anual se está reduciendo como lo detecta el cociente $\left(\mathrm{CO}_{15}\right)$ entre la lluvia media de los últimos 15 años y la de largo plazo, cuya media y moda son 0.978 y 0.975 , respectivamente.

\section{Metodología}

Estaciones pluviométricas procesadas

De inicio se estableció emplear solo estaciones pluviométricas con un periodo amplio de registro; se encontró conveniente, por disponibilidad, usar las de más de 50 años de registro. El archivo en Excel de la precipitación mensual de la Dirección Local Zacatecas de la Comisión Nacional del Agua (CONAGUA), cuenta con 133 estaciones pluviométricas. De ellas únicamente 22 tienen un registro mayor de 50 años, pero las estaciones Trancoso, Jeréz y Teúl de González Ortega, fueron eliminadas por tener muchos años incompletos; entonces se utilizaron las 19 mostradas en la tabla 1 , que no contienen datos en la columna 7 de dicha tabla.

Utilizando la información histórica contenida en los Boletines Climatológicos (SARH, 1977; 1980a; 1980b) para 11 estaciones más se logró completar sus registros, según se indica en la columna 7 de la tabla 1, integrando un total de 30 registros por procesar de precipitación anual en milímetros. En la figura 1 se muestra la ubicación geográfica de las 30 estaciones pluviométricas procesadas.

En la figura 1 se indican los parteaguas de las cuatro regiones hidrológicas que comprende el estado de Zacatecas. La región hidrológica núm. 11 de los Ríos Presidio y San Pedro que drenan al Océano Pacífico, abarca la porción menor en el oeste del estado, en esa zona únicamente se ubicó la estación Chalchihuites. En la región hidrológica núm. 12 Parcial del Río Santiago, que también fluye al Océano Pacífico, se ubican las zonas sur del estado y centro poniente; en esta región se procesaron 15 estaciones pluviométricas.

La región hidrológica núm. 36 de los ríos Nazas y Aguanaval es endorreica y estos confluyen a lo que era la Laguna de Mayrán, cerca de la Comarca Lagunera. Esta región comienza en la zona centro del estado y avanza hacia el norte con la cuenca del río Aguanaval y en ella se ubican las cinco estaciones pluviométricas analizadas. Otra porción de esta región se ubica en el norte del estado. Finalmente, la región hidrológica núm. 37 denominada El Salado que también es endorreica y está integrada por ríos y corrientes de poca importancia, comprende la porción norte y oriente del estado. En esta zona se procesaron nueve estaciones pluviométricas. 
Tabla 1. Características generales de las 30 estaciones pluviométricas procesadas del estado de Zacatecas, México

\begin{tabular}{|c|c|c|c|c|c|c|c|c|}
\hline \multirow[b]{2}{*}{ Núm. } & \multirow[b]{2}{*}{ Nombre } & \multirow{2}{*}{$\begin{array}{l}\text { Latitud } \\
\text { Norte }\end{array}$} & \multirow{2}{*}{$\begin{array}{l}\text { Longitud } \\
\text { W.G. }\end{array}$} & \multirow{2}{*}{$\begin{array}{l}\text { Altitud } \\
\text { m.s.n.m. }\end{array}$} & \multirow{2}{*}{$\begin{array}{l}\text { R. H. }{ }^{1} \\
\text { Núm. }\end{array}$} & \multicolumn{3}{|c|}{ Periodo del registro $\left(\mathrm{NA}^{2}\right)$} \\
\hline & & & & & & Boletín SARH & CONAGUA & Integrado \\
\hline 1 & Chalchihuites & $23^{\circ} 29^{\prime}$ & $103^{\circ} 52^{\prime}$ & 2260 & 11 & - & 1962-2012 (51) & $(51)$ \\
\hline 2 & El Cazadero & $23^{\circ} 42^{\prime}$ & $103^{\circ} 06^{\prime}$ & 1920 & 36 & - & 1958-2012 (55) & (55) \\
\hline 3 & $\begin{array}{c}\text { Leobardo } \\
\text { Reynoso (El Sauz) }\end{array}$ & $23^{\circ} 11^{\prime}$ & $103^{\circ} 12^{\prime}$ & 2090 & 36 & 1938-1946 (9) & 1947-2012 (66) & (75) \\
\hline 4 & Juan Aldama & $24^{\circ} 17^{\prime}$ & $103^{\circ} 23^{\prime}$ & 1995 & 36 & 1962-1974 (13) & 1975-2012 (38) & (51) \\
\hline 5 & Río Grande & $23^{\circ} 48^{\prime}$ & $103^{\circ} 02^{\prime}$ & 1890 & 36 & 1947-1965 (19) & $1975-2012(38)$ & $\left(66^{3}\right)$ \\
\hline 6 & Santa Rosa & $22^{\circ} 56^{\prime}$ & $103^{\circ} 07^{\prime}$ & 2240 & 36 & 1939-1946 (8) & $1947-2012(66)$ & (74) \\
\hline 7 & Agua Nueva & $23^{\circ} 47^{\prime}$ & $102^{\circ} 06^{\prime}$ & 1932 & 37 & - & $1963-2012(50)$ & (50) \\
\hline 8 & Calera & $22^{\circ} 54^{\prime}$ & $102^{\circ} 39^{\prime}$ & 2192 & 37 & - & 1958-2012 (55) & (55) \\
\hline 9 & $\begin{array}{l}\text { Concepción del } \\
\text { Oro }\end{array}$ & $24^{\circ} 37^{\prime}$ & $101^{\circ} 23^{\prime}$ & 1940 & 37 & 1951-1959 (8) & 1960-2012 (53) & (61) \\
\hline 10 & $\begin{array}{l}\text { Cañitas de Felipe } \\
\text { Pescador }\end{array}$ & $23^{\circ} 36^{\prime}$ & $102^{\circ} 44^{\prime}$ & 2025 & 37 & 1942-1973 (32) & 1974-2012 (39) & (71) \\
\hline 11 & Fresnillo & $23^{\circ} 11^{\prime}$ & $102^{\circ} 53^{\prime}$ & 2195 & 37 & - & 1949-2012 (64) & (64) \\
\hline 12 & Gruñidora & $24^{\circ} 16^{\prime}$ & $101^{\circ} 53^{\prime}$ & 1825 & 37 & - & $1963-2012(50)$ & $\left(50^{4}\right)$ \\
\hline 13 & Pinos & $22^{\circ} 17^{\prime}$ & $101^{\circ} 35^{\prime}$ & 2408 & 37 & - & 1947-2012 (66) & $\left(66^{3}\right)$ \\
\hline 14 & Villa de Cos & $23^{\circ} 17^{\prime}$ & $102^{\circ} 21^{\prime}$ & 2050 & 37 & 1942-1961 (20) & $1962-2012(51)$ & $(71)$ \\
\hline 15 & Zacatecas & $22^{\circ} 46^{\prime}$ & $102^{\circ} 35^{\prime}$ & 2485 & 37 & 1930-1952 (23) & $1953-2012(60)$ & $\left(83^{4}\right)$ \\
\hline 16 & El Platanito & $22^{\circ} 34^{\prime}$ & $104^{\circ} 03^{\prime}$ & 990 & 12 & - & 1957-2012 (56) & (56) \\
\hline 17 & Excamé & $21^{\circ} 39^{\prime}$ & $103^{\circ} 22^{\prime}$ & 1740 & 12 & - & 1946-2012 (67) & (67) \\
\hline 18 & Jiménez del Teúl & $23^{\circ} 15^{\prime}$ & $103^{\circ} 48^{\prime}$ & 1900 & 12 & - & 1963-2012 (50) & (50) \\
\hline 19 & Juchipila & $21^{\circ} 23^{\prime}$ & $103^{\circ} 07^{\prime}$ & 1270 & 12 & - & 1947-2012 (66) & (66) \\
\hline 20 & La Florida & $22^{\circ} 41^{\prime}$ & $103^{\circ} 36^{\prime}$ & 1870 & 12 & - & 1954-2012 (58) & (58) \\
\hline 21 & La Villita & $21^{\circ} 36^{\prime}$ & $103^{\circ} 20^{\prime}$ & 1790 & 12 & - & $1957-2012(56)$ & (56) \\
\hline 22 & Loreto & $22^{\circ} 17^{\prime}$ & $101^{\circ} 59^{\prime}$ & 2029 & 12 & - & 1963-2012 (50) & (50) \\
\hline 23 & Monte Escobedo & $22^{\circ} 20^{\prime}$ & $103^{\circ} 32^{\prime}$ & 2190 & 12 & 1957-1963 (7) & 1964-2012 (49) & (56) \\
\hline 24 & Nochistlán & $21^{\circ} 22^{\prime}$ & $102^{\circ} 51^{\prime}$ & 1850 & 12 & - & $1950-2012(63)$ & (63) \\
\hline 25 & Ojo Caliente & $22^{\circ} 25^{\prime}$ & $102^{\circ} 16^{\prime}$ & 2050 & 12 & - & 1961-2012 (52) & (52) \\
\hline 26 & Presa El Chique & $22^{\circ} 00^{\prime}$ & $102^{\circ} 53^{\prime}$ & 1620 & 12 & 1960-1963 (3) & 1951-2012 (59) & (62) \\
\hline 27 & $\begin{array}{c}\text { San Pedro Piedra } \\
\text { Gorda }\end{array}$ & $22^{\circ} 27^{\prime}$ & $102^{\circ} 21^{\prime}$ & 2032 & 12 & - & 1942-2012 (71) & (71) \\
\hline 28 & Tlaltenango & $21^{\circ} 47^{\prime}$ & $103^{\circ} 20^{\prime}$ & 1700 & 12 & - & 1950-2012 (63) & (63) \\
\hline 29 & Villa García & $22^{\circ} 10^{\prime}$ & $101^{\circ} 57^{\prime}$ & 2102 & 12 & - & 1950-2012 (63) & (63) \\
\hline 30 & Villanueva & $22^{\circ} 22^{\prime}$ & $102^{\circ} 53^{\prime}$ & 1920 & 12 & 1950-1962 (13) & $1963-2012(50)$ & (63) \\
\hline
\end{tabular}

${ }^{1}$ región hidrológica $\quad{ }^{2}$ número de años $\quad{ }^{3}$ se estimaron varios años por regresión $\quad{ }^{4}$ se transportó información de estación cercana

\section{Deducción de datos faltantes}

Con excepción de las estaciones pluviométricas Fresnillo y Leobardo Reynoso (El Sauz), el resto tienen registros de precipitación mensual incompletos, por ello se adoptó el proceso siguiente para su estimación. Cuando en un año incompleto faltaba un máximo de tres meses, estos se consideraron iguales a la media mensual. Si los tres meses faltantes se ubicaban en la época de lluvias (junio a septiembre) o bien eran más, se estimó el valor anual de la precipitación mediante regresión lineal con el registro más cercano.
Con este procedimiento se estimó el lapso faltante de 1966 a 1975 en la estación pluviométrica Río Grande, con base en los datos de El Cazadero, usando como periodo común 1975 a $2012(n=38)$ y suprimiendo seis valores dispersos para mejorar el coeficiente de correlación lineal $\left(r_{x y}\right)$ de 0.7536 a 0.8912 . Para estimar el lapso de 1987 a 1992 en la estación pluviométrica Pinos, no se pudieron utilizar los registros cercanos, pues también carecen de información, por ello se empleó la estación El Nigromante, con un periodo común de 22 datos en el lapso de 1984 a 2012; al suprimir dos valores dispersos el $r_{x y}$ resultó de 0.7083 . 


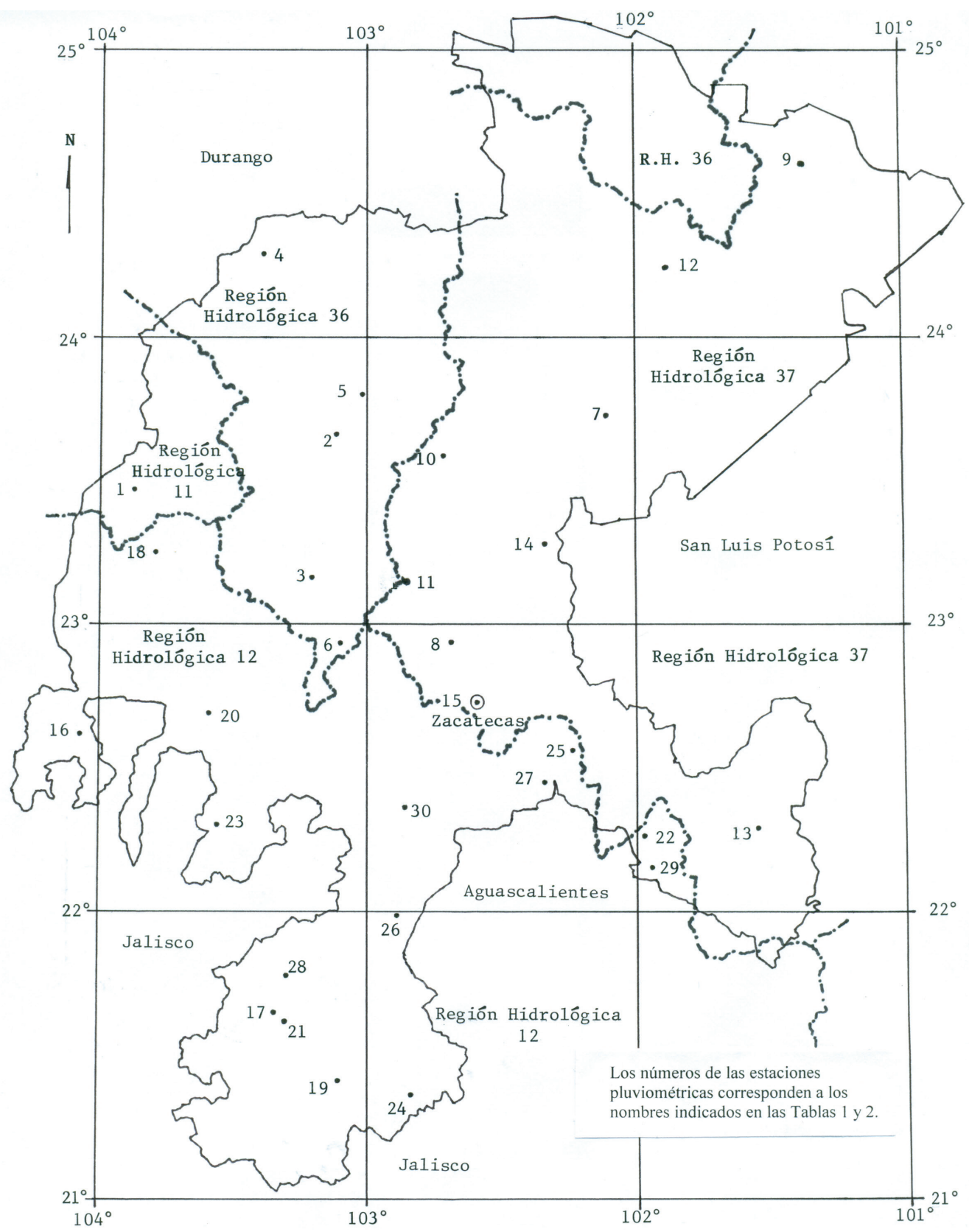

Figura 1. Localización geográfica de las 30 estaciones pluviométricas procesadas del estado de Zacatecas, México 
Para completar el registro incompleto de la estación pluviométrica Zacatecas en los años 2010 a 2012, los datos mensuales faltantes se consideraron iguales a los registrados en la estación Guadalupe, que es bastante cercana, pues dista menos de $8 \mathrm{~km}$ en línea recta y se ubica en la misma zona geográfica. Por último, en la estación pluviométrica Gruñidora los años faltantes 2008, 2011 y 2012 se dedujeron con proporcionalidad de medias anuales en el lapso 1995 a 2012, con base en los datos de la estación Nuevo Mercurio que es la más cercana.

\section{Pruebas estadísticas}

Requerimientos estadísticos de los registros

Todos los análisis estadísticos y probabilísticos realizados con los registros hidrológicos o climatológicos de datos anuales, se basan en una serie de requerimientos estadísticos básicos, como son que la serie o registro sea consistente, libre de tendencia, no periódica y que constituya un proceso estocástico, cuya componente aleatoria siga una determinada función de distribución de probabilidades. La consistencia implica que todos los datos medidos pertenecen a la misma población estadística y tienen por lo tanto una media invariante en el tiempo; esta propiedad también se conoce como homogeneidad y sus pruebas estadísticas buscan cambios significativos en la media.

La tendencia ocurre en los datos si existe una correlación significativa (positiva o negativa) entre los datos y el tiempo. La tendencia vuelve a la serie no estacionaria, es comúnmente introducida por los cambios en el uso del suelo o por el cambio climático. Finalmente, la aleatoriedad en un registro hidrológico o climatológico significa que los datos fluctúan exclusivamente por el fenómeno natural (lluvia o escurrimiento); cuando no hay aleatoriedad el registro es persistente. La persistencia se define como la propensión de los datos de una serie a recordar la magnitud de sus valores antecedentes o a ser influenciados por ellos. Debido a lo anterior, se cuantifica con base en el coeficiente de correlación serial que define la dependencia entre cada elemento $i \mathrm{y}$ el $i-k$; por ello $k$ se llama "tiempo de retraso" (Adeloye y Montaseri, 2002; Machiwal y Jha, 2008; 2012).

\section{Pruebas y tests aplicados}

Las pruebas estadísticas pueden ser paramétricas y no paramétricas; las segundas no están basadas en la consideración de que la población de los datos es normal, por ello se utilizan ampliamente, pero son pruebas que tienen menor potencia que los tests paramétricos. Se ha llegado a establecer que para obtener la misma conclusión con igual nivel de confianza, las pruebas no paramétricas requieren de 5 a 35\% más datos que los tests paramétricos (Machiwal y Jha, 2012).

La aplicación de las pruebas paramétricas requiere que los datos sigan una distribución normal, entonces primero se determinó con los tests del cociente de Geary (CG) y de Shapiro-Wilk (SW) que si para los registros procesados de precipitación anual se puede aceptar que provengan de una distribución normal. Los resultados de ambos tests se tienen en la tabla 2, las columnas 3 y 4 indican la normalidad con $\mathrm{N}$ y la no procedencia de una distribución normal con NN.

Las pruebas de Helmert (PH) y de las secuencias (PS) detectan si la serie o registro es homogéneo, debido a que no tiene persistencia o tendencia, cambio en la media, o bien, no oscila demasiado. En cambio, la prueba de Von Neumann (PV) es de aleatoriedad contra componentes determinísticas no especificadas. Los resultados de estas pruebas se exponen en las columnas 5 a 7 de la tabla 2 , con $\mathrm{H}$ para registros homogéneos y con $\mathrm{NH}$ para los no homogéneos.

Respecto a los tests específicos, se aplicaron los 11 siguientes:

1. Dos de persistencia, el de Anderson (TA) y el de Sneyers $\left(\mathrm{TS}_{1}\right)$.

2. Tres de tendencia, el de Kendall (TK), el de Spearman $\left(\mathrm{TS}_{2}\right)$ y el de Mann-Kendall (TM).

3. Uno de variabilidad, el de Bartlett (TB).

4. Uno de cambio en la media, el de Cramer (TC) y

5. Cuatro que buscan cambios en la media o pérdida de homogeneidad con estadísticos basados en la curva de masa residual $\left(\mathrm{TB}_{1}\right.$ a $\left.\mathrm{TB}_{4}\right)$.

Todas las pruebas se aplicaron con un nivel de significancia $(\alpha)$ de $5 \%$. En el apéndice 1 se citan las referencias donde se pueden consultar 13 de los tests anteriores, pues los tres relativos a la tendencia se exponen en el apéndice 2 . Los resultados de estos 11 tests específicos se muestran en las columnas 8 a 18 de la tabla 2, usando $\mathrm{H}$ cuando no detectan la componente que buscan y $\mathrm{NH}$ cuando la encuentran.

\section{Discusión de resultados}

\section{Pruebas estadísticas}

El test de Shapiro-Wilk detecta ocho registros que no proceden de una distribución normal. La correspondencia entre estos resultados y los valores del cociente 
de Geary (CG), permite establecer que cuando excede a 1.060 o es menor que 0.940 , la serie no presenta normalidad. Las diferencias encontradas en los resultados de ambos tests (tabla 2), ratifican que el CG es menos consistente, según señalan Machiwal y Jha (2012). Según valores de la tabla 4, cuando el coeficiente de asimetría (Cs) es mayor de 0.600 , el registro no procede de una distribución normal.
En la tabla 3 se citan las componentes determinísticas detectadas en 18 estaciones pluviométricas que mostraron anomalías según las 14 pruebas estadísticas aplicadas. En general, cuando la prueba de Helmert o la de las Secuencias muestran falta de homogeneidad, se debe a exceso de secuencias y se señala como "oscila poco". Cuando con la prueba de Von Neumann se encontró pérdida de homogeneidad, el registro se indicó

Tabla 2. Resultados de las pruebas estadísticas en las 30 estaciones pluviométricas procesadas, del estado de Zacatecas, México

\begin{tabular}{|c|c|c|c|c|c|c|c|c|c|c|c|c|c|c|c|c|c|}
\hline 1 & 2 & 3 & 4 & 5 & 6 & 7 & 8 & 9 & 10 & 11 & 12 & 13 & 14 & 15 & 16 & 17 & 18 \\
\hline \multirow[b]{2}{*}{ Núm } & \multirow[b]{2}{*}{ Nombre } & \multicolumn{2}{|c|}{ Normalidad } & \multicolumn{3}{|c|}{ Pruebas generales } & \multicolumn{11}{|c|}{ Tests específicos } \\
\hline & & CG & SW & $\mathrm{PH}$ & PS & PV & TA & $\mathrm{TS}_{1}$ & TK & $\mathrm{TS}_{2}$ & $\mathrm{TM}$ & ТВ & TC & $\mathrm{TB}_{1}$ & $\mathrm{~TB}_{2}$ & $\mathrm{~TB}_{3}$ & $\mathrm{~TB}_{4}$ \\
\hline 1 & Chalchihuites & 1.009 & $\mathrm{~N}$ & $\mathrm{NH}$ & $\mathrm{NH}$ & $\mathrm{H}$ & $\mathrm{NH}$ & $\mathrm{H}$ & $\mathrm{H}$ & $\mathrm{H}$ & $\mathrm{H}$ & $\mathrm{H}$ & $\mathrm{H}$ & $\mathrm{H}$ & $\mathrm{NH}$ & $\mathrm{H}$ & $\mathrm{H}$ \\
\hline 2 & El Cazadero & 0.983 & $\mathrm{~N}$ & $\mathrm{H}$ & $\mathrm{H}$ & $\mathrm{H}$ & $\mathrm{H}$ & $\mathrm{H}$ & $\mathrm{H}$ & $\mathrm{H}$ & $\mathrm{H}$ & $\mathrm{H}$ & $\mathrm{H}$ & $\mathrm{H}$ & $\mathrm{H}$ & $\mathrm{H}$ & $\mathrm{H}$ \\
\hline 3 & $\begin{array}{l}\text { Leobardo } \\
\text { Reynoso }\end{array}$ & 0.996 & $\mathrm{NN}$ & $\mathrm{H}$ & $\mathrm{H}$ & $\mathrm{H}$ & $\mathrm{H}$ & $\mathrm{NH}$ & $\mathrm{NH}$ & $\mathrm{NH}$ & $\mathrm{NH}$ & $\mathrm{H}$ & $\mathrm{H}$ & $\mathrm{H}$ & $\mathrm{H}$ & $\mathrm{H}$ & $\mathrm{H}$ \\
\hline 4 & Juan Aldama & 1.057 & $\mathrm{~N}$ & $\mathrm{H}$ & $\mathrm{H}$ & $\mathrm{H}$ & $\mathrm{H}$ & $\mathrm{H}$ & $\mathrm{H}$ & $\mathrm{H}$ & $\mathrm{H}$ & $\mathrm{H}$ & $\mathrm{H}$ & $\mathrm{H}$ & $\mathrm{H}$ & $\mathrm{H}$ & $\mathrm{H}$ \\
\hline 5 & Río Grande & 1.002 & $\mathrm{~N}$ & $\mathrm{NH}$ & $\mathrm{NH}$ & $\mathrm{H}$ & $\mathrm{H}$ & $\mathrm{H}$ & $\mathrm{H}$ & $\mathrm{H}$ & $\mathrm{H}$ & $\mathrm{H}$ & $\mathrm{H}$ & $\mathrm{H}$ & $\mathrm{H}$ & $\mathrm{H}$ & $\mathrm{H}$ \\
\hline 6 & Santa Rosa & 1.022 & $\mathrm{NN}$ & $\mathrm{NH}$ & $\mathrm{NH}$ & $\mathrm{NH}$ & $\mathrm{NH}$ & $\mathrm{NH}$ & $\mathrm{H}$ & $\mathrm{H}$ & $\mathrm{H}$ & $\mathrm{H}$ & $\mathrm{H}$ & $\mathrm{H}$ & $\mathrm{H}$ & $\mathrm{H}$ & $\mathrm{H}$ \\
\hline 7 & Agua Nueva & 0.982 & $\mathrm{~N}$ & $\mathrm{NH}$ & $\mathrm{NH}$ & $\mathrm{NH}$ & $\mathrm{NH}$ & $\mathrm{NH}$ & $\mathrm{H}$ & $\mathrm{H}$ & $\mathrm{H}$ & $\mathrm{NH}$ & $\mathrm{H}$ & $\mathrm{H}$ & $\mathrm{NH}$ & $\mathrm{H}$ & $\mathrm{H}$ \\
\hline 8 & Calera & 1.061 & $\mathrm{NN}$ & $\mathrm{NH}$ & $\mathrm{H}$ & $\mathrm{H}$ & $\mathrm{H}$ & $\mathrm{H}$ & $\mathrm{H}$ & $\mathrm{H}$ & $\mathrm{H}$ & $\mathrm{H}$ & $\mathrm{H}$ & $\mathrm{H}$ & $\mathrm{H}$ & $\mathrm{H}$ & $\mathrm{H}$ \\
\hline 9 & $\begin{array}{l}\text { Concepción del } \\
\text { Oro }\end{array}$ & 0.973 & $\mathrm{NN}$ & $\mathrm{NH}$ & $\mathrm{NH}$ & $\mathrm{NH}$ & $\mathrm{NH}$ & $\mathrm{NH}$ & $\mathrm{H}$ & $\mathrm{H}$ & $\mathrm{H}$ & $\mathrm{H}$ & $\mathrm{H}$ & $\mathrm{H}$ & $\mathrm{NH}$ & $\mathrm{H}$ & $\mathrm{H}$ \\
\hline 10 & Felipe Pescador & 0.978 & $\mathrm{NN}$ & $\mathrm{H}$ & $\mathrm{H}$ & $\mathrm{H}$ & $\mathrm{H}$ & $\mathrm{H}$ & $\mathrm{H}$ & $\mathrm{H}$ & $\mathrm{H}$ & $\mathrm{H}$ & $\mathrm{H}$ & $\mathrm{H}$ & $\mathrm{H}$ & $\mathrm{H}$ & $\mathrm{H}$ \\
\hline 11 & Fresnillo & 1.045 & $\mathrm{~N}$ & $\mathrm{NH}$ & $\mathrm{H}$ & $\mathrm{H}$ & $\mathrm{H}$ & $\mathrm{H}$ & $\mathrm{H}$ & $\mathrm{H}$ & $\mathrm{H}$ & $\mathrm{H}$ & $\mathrm{H}$ & $\mathrm{H}$ & $\mathrm{H}$ & $\mathrm{H}$ & $\mathrm{H}$ \\
\hline 12 & Gruñidora & 1.003 & $\mathrm{~N}$ & $\mathrm{NH}$ & $\mathrm{NH}$ & $\mathrm{NH}$ & $\mathrm{NH}$ & $\mathrm{NH}$ & $\mathrm{NH}$ & $\mathrm{NH}$ & $\mathrm{NH}$ & $\mathrm{H}$ & $\mathrm{NH}$ & $\mathrm{NH}$ & $\mathrm{NH}$ & $\mathrm{NH}$ & $\mathrm{NH}$ \\
\hline 13 & Pinos & 1.010 & $\mathrm{~N}$ & $\mathrm{H}$ & $\mathrm{H}$ & $\mathrm{H}$ & $\mathrm{H}$ & $\mathrm{H}$ & $\mathrm{H}$ & $\mathrm{H}$ & $\mathrm{H}$ & $\mathrm{H}$ & $\mathrm{H}$ & $\mathrm{H}$ & $\mathrm{H}$ & $\mathrm{H}$ & $\mathrm{H}$ \\
\hline 14 & Villa de Cos & 0.995 & $\mathrm{NN}$ & $\mathrm{H}$ & $\mathrm{H}$ & $\mathrm{H}$ & $\mathrm{H}$ & $\mathrm{H}$ & $\mathrm{H}$ & $\mathrm{H}$ & $\mathrm{H}$ & $\mathrm{H}$ & $\mathrm{H}$ & $\mathrm{H}$ & $\mathrm{H}$ & $\mathrm{H}$ & $\mathrm{H}$ \\
\hline 15 & Zacatecas & 1.014 & $\mathrm{~N}$ & $\mathrm{H}$ & $\mathrm{H}$ & $\mathrm{NH}$ & $\mathrm{NH}$ & $\mathrm{H}$ & $\mathrm{H}$ & $\mathrm{H}$ & $\mathrm{H}$ & $\mathrm{H}$ & $\mathrm{H}$ & $\mathrm{H}$ & $\mathrm{H}$ & $\mathrm{H}$ & $\mathrm{H}$ \\
\hline 16 & El Platanito & 1.032 & $\mathrm{~N}$ & $\mathrm{H}$ & $\mathrm{NH}$ & $\mathrm{NH}$ & $\mathrm{NH}$ & $\mathrm{NH}$ & $\mathrm{H}$ & $\mathrm{H}$ & $\mathrm{H}$ & $\mathrm{H}$ & $\mathrm{H}$ & $\mathrm{NH}$ & $\mathrm{NH}$ & $\mathrm{H}$ & $\mathrm{H}$ \\
\hline 17 & Excamé & 0.989 & $\mathrm{~N}$ & $\mathrm{H}$ & $\mathrm{H}$ & $\mathrm{H}$ & $\mathrm{H}$ & $\mathrm{H}$ & $\mathrm{H}$ & $\mathrm{H}$ & $\mathrm{H}$ & $\mathrm{H}$ & $\mathrm{H}$ & $\mathrm{H}$ & $\mathrm{H}$ & $\mathrm{H}$ & $\mathrm{H}$ \\
\hline 18 & Jiménez del Teúl & 1.017 & $\mathrm{~N}$ & $\mathrm{NH}$ & $\mathrm{NH}$ & $\mathrm{H}$ & $\mathrm{H}$ & $\mathrm{NH}$ & $\mathrm{H}$ & $\mathrm{H}$ & $\mathrm{H}$ & $\mathrm{H}$ & $\mathrm{H}$ & $\mathrm{H}$ & $\mathrm{H}$ & $\mathrm{H}$ & $\mathrm{H}$ \\
\hline 19 & Juchipila & 1.007 & $\mathrm{~N}$ & $\mathrm{H}$ & $\mathrm{H}$ & $\mathrm{H}$ & $\mathrm{H}$ & $\mathrm{H}$ & $\mathrm{H}$ & $\mathrm{H}$ & $\mathrm{H}$ & $\mathrm{H}$ & $\mathrm{H}$ & $\mathrm{H}$ & $\mathrm{H}$ & $\mathrm{H}$ & $\mathrm{H}$ \\
\hline 20 & La Florida & 0.997 & $\mathrm{~N}$ & $\mathrm{H}$ & $\mathrm{H}$ & $\mathrm{H}$ & $\mathrm{H}$ & $\mathrm{H}$ & $\mathrm{H}$ & $\mathrm{H}$ & $\mathrm{H}$ & $\mathrm{H}$ & $\mathrm{H}$ & $\mathrm{H}$ & $\mathrm{H}$ & $\mathrm{H}$ & $\mathrm{H}$ \\
\hline 21 & La Villita & 0.975 & $\mathrm{~N}$ & $\mathrm{H}$ & $\mathrm{H}$ & $\mathrm{H}$ & $\mathrm{H}$ & $\mathrm{H}$ & $\mathrm{H}$ & $\mathrm{H}$ & $\mathrm{H}$ & $\mathrm{H}$ & $\mathrm{H}$ & $\mathrm{H}$ & $\mathrm{H}$ & $\mathrm{H}$ & $\mathrm{H}$ \\
\hline 22 & Loreto & 0.965 & $\mathrm{~N}$ & $\mathrm{H}$ & $\mathrm{H}$ & $\mathrm{H}$ & $\mathrm{H}$ & $\mathrm{H}$ & $\mathrm{H}$ & $\mathrm{H}$ & $\mathrm{H}$ & $\mathrm{H}$ & $\mathrm{H}$ & $\mathrm{H}$ & $\mathrm{H}$ & $\mathrm{H}$ & $\mathrm{H}$ \\
\hline 23 & Monte Escobedo & 0.968 & $\mathrm{NN}$ & $\mathrm{NH}$ & $\mathrm{NH}$ & $\mathrm{H}$ & $\mathrm{H}$ & $\mathrm{NH}$ & $\mathrm{H}$ & $\mathrm{H}$ & $\mathrm{H}$ & $\mathrm{NH}$ & $\mathrm{H}$ & $\mathrm{H}$ & $\mathrm{H}$ & $\mathrm{H}$ & $\mathrm{H}$ \\
\hline 24 & Nochistlán & 0.987 & $\mathrm{~N}$ & $\mathrm{NH}$ & $\mathrm{H}$ & $\mathrm{H}$ & $\mathrm{H}$ & $\mathrm{H}$ & $\mathrm{H}$ & $\mathrm{H}$ & $\mathrm{H}$ & $\mathrm{H}$ & $\mathrm{H}$ & $\mathrm{H}$ & $\mathrm{H}$ & $\mathrm{H}$ & $\mathrm{H}$ \\
\hline 25 & Ojo Caliente & 1.013 & $\mathrm{~N}$ & $\mathrm{H}$ & $\mathrm{H}$ & $\mathrm{NH}$ & $\mathrm{NH}$ & $\mathrm{NH}$ & $\mathrm{H}$ & $\mathrm{H}$ & $\mathrm{H}$ & $\mathrm{H}$ & $\mathrm{H}$ & $\mathrm{H}$ & $\mathrm{NH}$ & $\mathrm{H}$ & $\mathrm{H}$ \\
\hline 26 & Presa El Chique & 1.057 & $\mathrm{~N}$ & $\mathrm{H}$ & $\mathrm{NH}$ & $\mathrm{H}$ & $\mathrm{H}$ & $\mathrm{NH}$ & $\mathrm{H}$ & $\mathrm{H}$ & $\mathrm{H}$ & $\mathrm{H}$ & $\mathrm{H}$ & $\mathrm{H}$ & $\mathrm{H}$ & $\mathrm{H}$ & $\mathrm{H}$ \\
\hline 27 & $\begin{array}{c}\text { San Pedro Piedra } \\
\text { Gorda }\end{array}$ & 0.991 & $\mathrm{~N}$ & $\mathrm{H}$ & $\mathrm{H}$ & $\mathrm{NH}$ & $\mathrm{NH}$ & $\mathrm{H}$ & $\mathrm{H}$ & $\mathrm{H}$ & $\mathrm{H}$ & $\mathrm{H}$ & $\mathrm{H}$ & $\mathrm{H}$ & $\mathrm{H}$ & $\mathrm{H}$ & $\mathrm{H}$ \\
\hline 28 & Tlaltenango & 0.942 & $\mathrm{NN}$ & $\mathrm{H}$ & $\mathrm{H}$ & $\mathrm{H}$ & $\mathrm{H}$ & $\mathrm{H}$ & $\mathrm{H}$ & $\mathrm{H}$ & $\mathrm{H}$ & $\mathrm{H}$ & $\mathrm{H}$ & $\mathrm{H}$ & $\mathrm{H}$ & $\mathrm{H}$ & $\mathrm{H}$ \\
\hline 29 & Villa García & 1.058 & $\mathrm{~N}$ & $\mathrm{H}$ & $\mathrm{H}$ & $\mathrm{H}$ & $\mathrm{H}$ & $\mathrm{H}$ & $\mathrm{H}$ & $\mathrm{H}$ & $\mathrm{H}$ & $\mathrm{H}$ & $\mathrm{H}$ & $\mathrm{H}$ & $\mathrm{H}$ & $\mathrm{H}$ & $\mathrm{H}$ \\
\hline 30 & Villanueva & 1.033 & $\mathrm{~N}$ & $\mathrm{NH}$ & $\mathrm{NH}$ & NH & $\mathrm{NH}$ & $\mathrm{NH}$ & $\mathrm{H}$ & $\mathrm{H}$ & $\mathrm{H}$ & $\mathrm{H}$ & $\mathrm{H}$ & $\mathrm{H}$ & $\mathrm{H}$ & $\mathrm{H}$ & $\mathrm{H}$ \\
\hline
\end{tabular}


Tabla 3. Relación de estaciones pluviométricas que mostraron componentes determinísticas y su descripción

\begin{tabular}{|c|c|c|}
\hline Núm. & Nombre de la estación & Componentes determinísticas detectadas \\
\hline 1 & Chalchihuites & Oscila poco, persistencia ligera \\
\hline 2 & Leobardo Reynoso & Persistencia ligera y tendencia \\
\hline 3 & Río Grande & Oscila poco \\
\hline 4 & Santa Rosa & Oscila poco y persistencia \\
\hline 5 & Agua Nueva & $\begin{array}{l}\text { Oscila poco, persistencia y exceso de variabilidad (TB } \\
\text { y } \mathrm{TB}_{2} \text { ) por valor muy bajo de precipitación }(38.0 \mathrm{~mm} \\
\text { en } 1987 \text { ) }\end{array}$ \\
\hline 6 & Calera & Oscila poco \\
\hline 7 & Concepción del Oro & $\begin{array}{l}\text { Oscila poco, no aleatorio, persistencia y cambio en la } \\
\text { media }\left(\mathrm{TB}_{2}\right) \text { por valor muy bajo de lluvia }(95.7 \mathrm{~mm} \text { en } \\
\text { 1960) }\end{array}$ \\
\hline 8 & Fresnillo & Oscila poco \\
\hline 9 & Gruñidora & Persistencia, tendencia y cambio en la media \\
\hline 10 & Zacatecas & No aleatorio y persistencia ligera \\
\hline 11 & El Platanito & $\begin{array}{l}\text { Oscila poco, no aleatorio, persistencia y cambio en la } \\
\text { media }\left(\mathrm{TB}_{1} \text { y } \mathrm{TB}_{2} \text { ) por valor muy bajo de lluvia ( } 261.8\right. \\
\text { mm en 2011) }\end{array}$ \\
\hline 12 & Jiménez del Teúl & Oscila poco, persistencia ligera \\
\hline 13 & Monte Escobedo & $\begin{array}{l}\text { Oscila poco, persistencia ligera y exceso de } \\
\text { variabilidad (TB) por valores altos de lluvia (1197.5 } \\
\text { mm en } 1958 \text { y } 1242.7 \text { en 1963) }\end{array}$ \\
\hline 14 & Nochistlán & Oscila poco \\
\hline 15 & Ojo Caliente & No aleatorio y persistencia \\
\hline 16 & Presa El Chique & Oscila poco, persistencia ligera \\
\hline 17 & San Pedro Piedra Gorda & Oscila poco, persistencia ligera \\
\hline 18 & Villanueva & Oscila poco, no aleatorio y persistencia \\
\hline
\end{tabular}

como "no aleatorio". Por último, cuando la persistencia se detectó únicamente con alguna de las dos pruebas, se señaló como "persistencia ligera".

La persistencia puede considerarse una característica estadística del registro que se procesa, la cual se debe tomar en cuenta al realizar el análisis estocástico de la serie, a través de los modelos ARMA (Adeloye y Montaseri, 2002; Machiwal y Jha, 2008). Por lo anterior, todas las estaciones pluviométricas que mostraron esta característica no se eliminan. En las figuras 2, 3 y 4 se muestra el comportamiento de los registros de las estaciones Leobardo Reynoso, Gruñidora y El Platanito, las dos primeras presentaron tendencia y la tercera, cambio en la media, según las pruebas $\mathrm{TB}_{1}$ y $\mathrm{TB}_{2}$. La estación Gruñidora solo resultó homogénea en el test de Bartlett. Es importante destacar que cada una de estas tres estaciones pluviométricas que resultaron no homogéneas, pertenecen a las regiones hidrológicas números 36,37 y 12, respectivamente; indicando con ello que todos los ambientes geográficos del estado de Zacatecas son susceptibles de esas anomalías.
En estos registros se ajustó un modelo lineal para la tendencia y se buscó si su pendiente resultaba estadísticamente diferente de cero con una prueba basada en la distribución $t$ de Student, únicamente en la estación Gruñidora resultó significativa, pero en Leobardo Reynoso quedó en el límite, pues su estadístico alcanzó un valor de 1.980 y el crítico es 1.993 , con $\alpha=5 \%$. La descripción de esta prueba se presenta en el apéndice 3. Tomando en cuenta estos resultados y los de la tabla 3, se consideró necesario eliminar las estaciones Leobardo Reynoso, Gruñidora y El Platanito.

\section{Cociente de precipitaciones medias}

Hu y Liu (2010) analizaron 31 registros de precipitación anual de periodo amplio (más de 50 años) a través de China, cuyo valor medio oscila de menos de $50 \mathrm{~mm}$ a más de 2000 mm, es decir, abarcan desde los climas áridos hasta los húmedos; encontrando que el cociente de la lluvia reciente (últimos 10 años) a la de largo plazo fluctuó de 0.89 a 1.11, con un valor medio igual a la uni- 
dad. Por ello, concluyen que la lluvia en tal país no muestra efectos del cambio climático.

La tabla 4 se forma por tres partes, en la primera se exponen los parámetros estadísticos de la precipitación anual: media $(\bar{X})$, desviación estándar $(S)$ y coeficientes de variación $(C v)$, asimetría $(C s)$, curtosis $(C k)$ y correlación serial de orden uno $\left(r_{1}\right)$. En la segunda parte, se tienen las medias $\left(\bar{x}_{i}\right)$ de los últimos 5,10 y 15 años del registro y en la tercera los cocientes de $\bar{x}_{i}$ entre $\bar{X}$.

Los últimos dos renglones de la tabla 4 muestran que los valores de la media y de la moda de los tres cocientes son casi iguales, con una moda ligeramente me- nor. Lo anterior implica que los 27 valores calculados de cada cociente no están sesgados, indicando que varían poco, como lo demuestran sus magnitudes mínima y máxima. El cociente $\mathrm{CO}_{5}$ es el más bajo, debido a la presencia de la sequía de los años 2010 a 2012. El cociente $\mathrm{CO}_{10}$ es cercano a la unidad y además en 16 de los 27 valores calculados, excede la unidad. Desafortunadamente, el cociente $\mathrm{CO}_{15}$ tiene una media y moda menores que la unidad (0.978 y 0.975) y solo ocurren siete con magnitud mayor que uno y seis de ellos no exceden 1.10. La moda se estimó con base en la distribución gamma mixta.

Tabla 4. Parámetros estadísticos y cocientes de medias de la precipitación anual en las 27 estaciones pluviométricas de amplio registro del estado de Zacatecas, México

\begin{tabular}{|c|c|c|c|c|c|c|c|c|c|c|c|c|c|}
\hline \multirow[b]{2}{*}{ Núm. } & \multirow[b]{2}{*}{ Nombre } & \multicolumn{6}{|c|}{ Parámetros estadísticos } & \multicolumn{3}{|c|}{ Medias } & \multicolumn{3}{|c|}{ Cocientes $\left(\bar{x}_{i} / \bar{X}\right)$} \\
\hline & & $\bar{X}$ & $S$ & $C v$ & Cs & $C k$ & $r_{1} \bar{x}_{5}$ & $\bar{x}_{10}$ & $\bar{x}_{15}$ & & $\mathrm{CO}_{5}$ & $\mathrm{CO}_{10}$ & $\mathrm{CO}_{15}$ \\
\hline 1 & Chalchihuites & 529.8 & 166.4 & 0.314 & -0.315 & 2.694 & 0.220 & 528.5 & 596.4 & 564.6 & 0.998 & 1.126 & 1.066 \\
\hline 2 & El Cazadero & 403.5 & 125.0 & 0.310 & 0.491 & 3.459 & 0.105 & 355.6 & 443.4 & 408.6 & 0.881 & 1.099 & 1.013 \\
\hline 3 & Juan Aldama & 427.8 & 121.1 & 0.283 & 0.362 & 2.204 & -0.041 & 427.5 & 475.6 & 436.9 & 0.999 & 1.112 & 1.021 \\
\hline 4 & Río Grande & 382.9 & 126.3 & 0.330 & 0.461 & 3.303 & -0.034 & 354.2 & 393.7 & 379.2 & 0.925 & 1.028 & 0.991 \\
\hline 5 & Santa Rosa & 452.3 & 143.4 & 0.317 & 0.668 & 3.682 & 0.194 & 398.0 & 474.5 & 474.2 & 0.880 & 1.049 & 1.048 \\
\hline 6 & Agua Nueva & 330.1 & 140.6 & 0.426 & 0.682 & 3.571 & 0.250 & 410.1 & 390.0 & 354.7 & 1.242 & 1.182 & 1.075 \\
\hline 7 & Calera & 429.0 & 137.2 & 0.320 & 0.557 & 2.717 & 0.122 & 350.2 & 444.1 & 421.8 & 0.816 & 1.035 & 0.983 \\
\hline 8 & Concepción del Oro & 418.5 & 163.9 & 0.392 & 0.832 & 5.164 & 0.243 & 327.7 & 402.5 & 368.5 & 0.783 & 0.962 & 0.880 \\
\hline 9 & Felipe Pescador & 375.3 & 125.4 & 0.334 & 0.954 & 5.176 & -0.134 & 311.1 & 364.5 & 359.2 & 0.829 & 0.971 & 0.957 \\
\hline 10 & Fresnillo & 411.3 & 120.1 & 0.292 & 0.222 & 2.448 & 0.100 & 357.9 & 432.0 & 406.3 & 0.870 & 1.050 & 0.988 \\
\hline 11 & Pinos & 447.4 & 149.0 & 0.333 & 0.306 & 2.734 & 0.103 & 454.1 & 470.8 & 427.3 & 1.015 & 1.052 & 0.955 \\
\hline 12 & Villa de Cos & 422.2 & 159.6 & 0.378 & 0.780 & 3.866 & 0.055 & 362.2 & 421.4 & 385.0 & 0.858 & 0.998 & 0.912 \\
\hline 13 & Zacatecas & 472.9 & 139.8 & 0.296 & 0.374 & 3.205 & 0.181 & 404.3 & 458.8 & 461.2 & 0.855 & 0.970 & 0.975 \\
\hline 14 & Excamé & 733.1 & 153.1 & 0.209 & -0.177 & 2.988 & 0.089 & 632.5 & 720.0 & 683.0 & 0.863 & 0.982 & 0.932 \\
\hline 15 & Jiménez del Teúl & 473.8 & 127.2 & 0.269 & 0.064 & 3.535 & 0.171 & 435.3 & 454.5 & 448.8 & 0.919 & 0.959 & 0.947 \\
\hline 16 & Juchipila & 694.8 & 142.5 & 0.205 & 0.038 & 3.301 & 0.077 & 583.5 & 663.1 & 666.2 & 0.840 & 0.954 & 0.959 \\
\hline 17 & La Florida & 582.9 & 142.6 & 0.245 & 0.235 & 2.808 & 0.119 & 554.3 & 611.4 & 572.0 & 0.951 & 1.049 & 0.981 \\
\hline 18 & La Villita & 771.7 & 178.7 & 0.232 & 0.570 & 3.657 & 0.139 & 625.8 & 730.3 & 726.7 & 0.811 & 0.946 & 0.942 \\
\hline 19 & Loreto & 442.1 & 124.6 & 0.282 & -0.505 & 3.691 & 0.011 & 397.6 & 435.2 & 415.2 & 0.899 & 0.984 & 0.939 \\
\hline 20 & Monte Escobedo & 717.4 & 169.1 & 0.236 & 0.831 & 4.391 & 0.059 & 681.0 & 738.0 & 693.3 & 0.949 & 1.029 & 0.966 \\
\hline 21 & Nochistlán & 699.9 & 164.6 & 0.235 & 0.006 & 3.061 & 0.065 & 594.7 & 644.1 & 610.8 & 0.850 & 0.920 & 0.873 \\
\hline 22 & Ojo Caliente & 390.8 & 141.7 & 0.363 & -0.057 & 2.788 & 0.337 & 436.4 & 442.7 & 407.7 & 1.117 & 1.133 & 1.043 \\
\hline 23 & Presa El Chique & 541.5 & 118.2 & 0.218 & -0.021 & 2.459 & 0.160 & 487.5 & 552.1 & 532.5 & 0.900 & 1.020 & 0.983 \\
\hline 24 & San Pedro Piedra Gorda & 415.4 & 136.6 & 0.329 & 0.207 & 2.755 & 0.186 & 364.6 & 418.6 & 401.5 & 0.878 & 1.008 & 0.967 \\
\hline 25 & Tlaltenango & 699.4 & 158.3 & 0.226 & 0.606 & 3.917 & 0.020 & 651.9 & 676.7 & 644.7 & 0.932 & 0.968 & 0.922 \\
\hline 26 & Villa García & 441.2 & 126.4 & 0.286 & 0.046 & 2.227 & -0.037 & 474.6 & 507.8 & 488.0 & 1.076 & 1.151 & 1.106 \\
\hline 27 & Villanueva & 466.5 & 145.9 & 0.313 & 0.213 & 2.254 & 0.399 & 423.8 & 498.1 & 459.9 & 0.908 & 1.068 & 0.986 \\
\hline \multicolumn{2}{|c|}{ Valores mínimos } & & - & - & - & - & - & - & - & - & 0.783 & 0.920 & 0.873 \\
\hline \multicolumn{2}{|c|}{ Valores máximos } & & - & - & - & - & - & - & - & - & 1.242 & 1.182 & 1.106 \\
\hline \multicolumn{2}{|c|}{ Valores promedio } & & - & - & - & - & - & - & - & - & 0.920 & 1.030 & 0.978 \\
\hline \multicolumn{2}{|c|}{ Valor de la moda } & & - & - & - & - & - & - & - & - & 0.910 & 1.025 & 0.975 \\
\hline
\end{tabular}



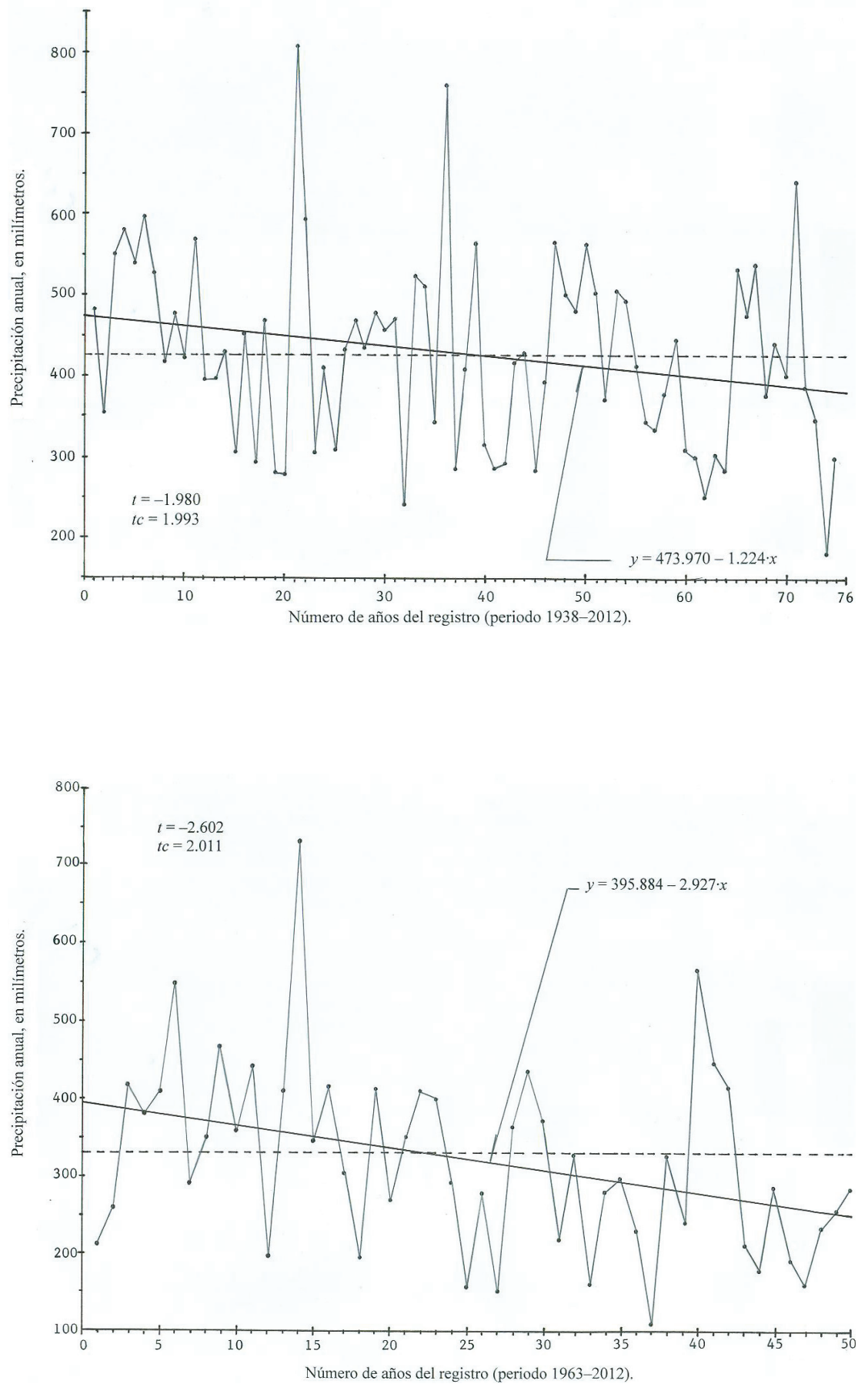

Figura 2. Serie cronológica de la precipitación anual $(\mathrm{mm})$ de la estación pluviométrica Leobardo Reynoso, Zacatecas, México
Figura 3. Serie cronológica de la precipitación anual $(\mathrm{mm})$ de la estación pluviométrica Gruñidora, Zacatecas, México 


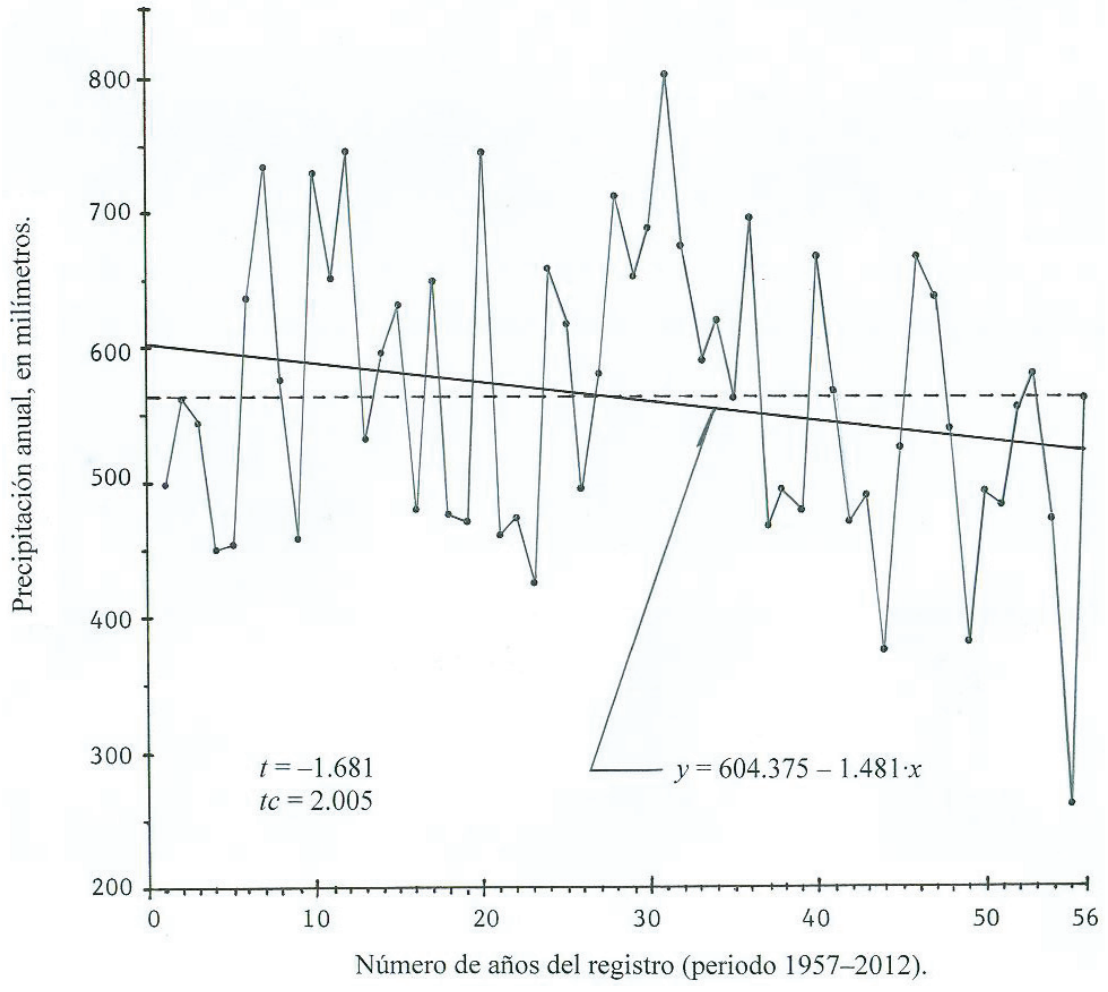

Figura 4. Serie cronológica de la precipitación anual $(\mathrm{mm})$ de la estación pluviométrica El Platanito, Zacatecas, México

\section{Conclusiones}

El resultado relevante de este estudio, es que la precipitación anual del estado de Zacatecas, México, está disminuyendo, pues el cociente $\mathrm{CO}_{15}$ fue menor que la unidad en 20 de los 27 registros procesados y donde excedió a uno, la mayoría no llegó a 1.10; además la media y la moda fueron semejantes con los valores siguientes: 0.978 y 0.975 , respectivamente.

Por otra parte, la comparación entre los tres cocientes $\left(\mathrm{CO}_{5}, \mathrm{CO}_{10} \mathrm{y} \mathrm{CO} \mathrm{CO}_{15}\right)$, conduce a detectar la presencia de una sequía importante en los últimos 5 años, ya que el $\mathrm{CO}_{5}$ es el más bajo con un valor medio de 0.92. Como el $\mathrm{CO}_{10}$ se recupera a un valor medio de 1.030, se intuye que la sequía citada tuvo una duración breve.

Aunque estadísticamente no existe evidencia general del cambio climático, pues únicamente dos registros de los 30 procesados, mostraron tendencia (estaciones pluviométricas Leobardo Reynoso y Gruñidora), esta fue decreciente en ambos y significativa en la estación Gruñidora y casi diferente de cero en la segunda (figuras 2 y 3). Los cocientes $\mathrm{CO}_{15}$ de las tres estaciones eliminadas (las dos anteriores y El Platanito), son respectivamente los siguientes: $0.901,0.858$ y 0.888 .

En la estación pluviométrica El Platanito se han medido en los últimos 15 años, los tres valores más bajos de precipitación de todo su registro de 56 años (figura 4). Esto influye, para ser detectado por dos pruebas basadas en la curva masa residual $\left(\mathrm{TB}_{1}\right.$ y $\left.\mathrm{TB}_{2}\right)$ y encontrar cambio en la media o pérdida de homogeneidad en tal registro.

Es importante señalar que las estaciones pluviométricas que resultaron no homogéneas, pertenecen a las regiones hidrológicas 36,37 y 12 , por esto se intuye que todos los ambientes geográficos del estado de Zacatecas son susceptibles de tales anomalías.

Se sugiere realizar este análisis en otros estados de la República Mexicana, o bien, en otras regiones o subregiones hidrológicas, para establecer si esta disminución de la precipitación es generalizada y donde se encuentre, como en el estado de Zacatecas, México, formular medidas orientadas a considerar esa reducción de lluvia y redimensionar los aprovechamientos hidráulicos de todo tipo.

\section{Agradecimientos}

Se agradecen las observaciones y correcciones sugeridas por el árbitro anónimo A, las cuales permitieron mejorar el enfoque general del trabajo y ayudaron a destacar sus resultados prácticos. 


\section{Apéndice 1}

Referencias de consulta de las pruebas estadísticas aplicadas

\begin{tabular}{lcl}
\hline \multicolumn{1}{c}{ Prueba o Test } & Símbolo & \multicolumn{1}{c}{ Referencias } \\
\hline Cociente de Geary & CG & Machiwal y Jha (2012) \\
Shapiro-Wilk & SW & Shapiro y Wilk (1965), Ruiz (1977) y Shapiro (1998) \\
Helmert & PH & Mather (1977) y Campos (1998) \\
Secuencias & PS & Thom (1971) y Campos (1998) \\
Von Neumann & PV & WMO (1971), Buishand (1982) y Machiwal y Jha (2012) \\
Anderson & TA & WMO (1971), Linsley et al. (1988) y Campos (1998) \\
Sneyers & TS & WMO (1971) \\
Bartlett & TB & WMO (1971) y Ruiz (1977) \\
Cramer & TC & WMO (1971) y Campos (1998) \\
Buishand & TB $_{1}$ a TB & Buishand (1982), Gutiérrez et al. (2007) y Campos (2008) \\
\hline
\end{tabular}

\section{Apéndice 2}

\section{Descripción de los Tests de Tendencia}

Machiwal y Jha (2008) aplicaron 12 pruebas para detección de tendencia, la mayoría paramétricas y encontraron que los tres tests que serán expuestos, fueron los de mayor potencia o más confiables.

\section{Test de Kendall (TK)}

Esta prueba paramétrica también se conoce como de correlación de rangos (Machiwal y Jha, 2012), es muy efectiva si la tendencia fundamental es de tipo lineal o se aproxima a esta. Su procedimiento consiste en evaluar el número $p$, en todos los pares de observaciones $\left(x_{i}, x_{j} ;\right.$ con $i=1$ a $n-1 ; j=i+1$ a $n$ ) en que $x_{j}$ es mayor que $x_{i \prime}$ después se calculan las estadísticas siguientes (Kottegoda, 1980):

$\tau=\left[\frac{4 p}{n(n-1)}\right]-1$

$\sigma_{\tau}^{2}=\frac{2(2 n+5)}{9 n(n-1)}$

Si el cociente $\tau / \sigma_{\tau}$ es menor que \pm 1.960 , el registro no presenta tendencia en una prueba de dos colas con un nivel de significancia $\alpha$ de $5 \%$.
Test de Spearman $\left(\mathrm{TS}_{2}\right)$

En esta prueba no paramétrica que se conoce como correlación del orden de rangos (Machiwal y Jha, 2012), primero se ordenan los datos de menor a mayor y se sustituye cada elemento del registro por su rango $\left(k_{i}\right)$, después para cada dato de la serie original se calculan las diferencias: $d_{i}=k_{i}-i$, con $i=1$ a $n$ y se evalúan las estadísticas siguientes (WMO, 1971; Adeloye y Montaseri, 2002):

$$
\begin{aligned}
& R_{k}=1-\frac{6 \sum_{i=1}^{n} d_{i}^{2}}{n\left(n^{2}-1\right)} \\
& t=R_{k} \sqrt{\frac{n-2}{1-R_{k}^{2}}}
\end{aligned}
$$

El valor de la ecuación anterior se compara con el intervalo que define $\pm t_{c}$ tomado de la distribución $t$ de Student con $n-2$ grados de libertad y nivel de significancia $\alpha$, para una prueba de dos colas; si lo excede $\left(t>t_{c}\right)$ existe tendencia.

Test de Mann-Kendall (TM)

Es una prueba no paramétrica que busca tendencia en la serie sin importar si es lineal o no lineal; pero el registro no debe mostrar persistencia. Su estadístico operati- 
vo $S$ s es la suma de los signos de las diferencias (z) de todas las parejas factibles de formar, por ello su expresión es (Manly, 2001):

$$
S S=\sum_{i=2}^{n} \sum_{j=1}^{i-1} \operatorname{signo}\left(x_{i}-x_{j}\right)
$$

en la cual, el signo(z) es -1 para $z<0,0$ para $z=0 y+1$ para $z>0$. Para una serie con valores aleatorios se espera que $S s=0$, cuya varianza es (Hirsch et al., 1993; Machiwal y Jha, 2012):

$\operatorname{Var}\left(S_{s}\right)=\frac{n(n-1)(2 n+5)}{18}$

El estadístico de la prueba es

$$
Z_{S}=\frac{S s+m_{c}}{\sqrt{\operatorname{Var}(S s)}}
$$

En la expresión anterior, $m_{c}=1$ cuando $S s<0$ y $m_{c}=-1$ para $S_{S}>0$. Si el valor absoluto de $Z_{S}$ es mayor que el valor crítico de la distribución normal estándar, la serie presenta tendencia creciente o decreciente con un nivel de significancia $\alpha$. Para $\alpha=5 \%$ el valor crítico es 1.960 . La varianza (ecuación 6) se debe corregir cuando existen datos iguales o la muestra es censurada (Hirsch et al., 1993).

\section{Apéndice 3}

Recta de regresión para una tendencia lineal

Se considera que la variable dependiente $(y)$ representa las precipitaciones anuales $P_{i}$ en milímetros y los tiempos o años $T_{i}$ son las abscisas $(x)$, en este caso iguales al $i$-ésimo valor $i$. Para probar si la pendiente $(m)$ de la recta de regresión ajustada por mínimos cuadrados de los residuos, es estadísticamente diferente de cero, se usa una prueba basada en la $t$ de Student definida por las ecuaciones siguientes (Ostele y Mensing, 1975):

$P=b+m \cdot T$

$b=\bar{P}-m \cdot \bar{T}$

$m=\frac{\frac{1}{n} \sum_{i=1}^{n} P_{i} \cdot i-\bar{P} \cdot \bar{T}}{\frac{1}{n} \sum_{i=1}^{n} i^{2}-\bar{T}^{2}}$
$t=\frac{m}{\sqrt{S_{m}^{2}}}$

$S_{m}^{2}=\frac{S_{E}^{2}}{\sum_{i=1}^{n}\left(T_{i}-\bar{T}\right)^{2}}$

$S_{E}^{2}=\frac{\sum_{i=1}^{n}\left(P_{i}-\hat{P}_{i}\right)^{2}}{(n-2)}$

$\bar{P}$ y $\bar{T}$ son las medias; en la ecuación anterior, $\hat{P}_{i}$ es el valor estimado con la recta de regresión (ecuación 8). $S_{E}^{2}$ y $S_{m}^{2}$ son las varianzas de los errores y de la pendiente. Si el valor calculado $t$ es mayor que el crítico $\left(t_{c}\right)$, obtenido para la distribución $t$ de Student con $n-2$ grados de libertad y $\alpha=5 \%$, en una prueba de dos colas, la pendiente $m$ es significativa, es decir, existe tendencia lineal. El problema de esta prueba es que no distingue entre persistencia y tendencia (Adeloye y Montaseri, 2002).

\section{Referencias}

Adeloye A.J. y Montaseri M. Preliminary streamflow data analyses prior to water resources study. Hydrological Sciences Journal, volumen 47 (número 5), 2002: 679-692.

Buishand T.A. Some methods for testing the homogeneity of rainfall records. Journal of Hydrology, volumen 58, 1982: 11-27.

Campos-Aranda D.F. Procesos del ciclo hidrológico, capítulo 4: precipitación, pp. 4.1-4.100 y capítulo 8: escurrimiento, pp. 8.18.76, $3^{\text {a }}$ reimpresión, San Luis Potosí, editorial Universitaria Potosina,1998.

Campos-Aranda D.F. Cuatro pruebas de homogeneidad para registros de lluvia anual basadas en la curva masa residual. XX Congreso Nacional de Hidráulica, tema: Hidrología superficial y subterránea, ponencia 6, octubre de 2008, Toluca, Estado de México.

Hirsch R.M., Helsel D.R., Cohn T.A., Gilroy E.J. Statistical Analysis of Hydrologic Data, capítulo 17, pp. 17.1-17.55, en: Handbook of Hydrology, editor in chief David R. Maidment, Nueva York, McGraw-Hill, Inc., 1993.

Gutiérrez-López A., Arellano-Lara F., Aparicio-Mijares F.J. Determinación de periodos de cambio climático, empleando pruebas de ruptura de series. Tláloc, (número 38), septiembre-enero de 2007: 4-11.

$\mathrm{Hu} \mathrm{Ch}$. y Liu Ch. Climate changes and changes of runoff and sediment loads from Chinese rivers. Climate change and adaptation for water resources in Yellow river basin, IHP, VII Technical Document in Hydrology, UNESCO office in Beijing, China, 2010, pp. 40-50. 
Kottegoda N.T. Stochastic water resources technology, capítulo 2: analysis of hydrologic time series, pp. 20-66, Londres, Inglaterra, MacMillan Press, Ltd, 1980, 384 p.

Kundzewicz Z.W. y Robson A.J. Change detection in hydrological records-A review of the methodology. Hydrological Sciences Journal, volumen 49 (número 1), febrero 2004: 7-18.

Linsley R.K., Kohler M.A., Paulhus J.L. Hydrology for Engineers, capítulo 14: Stochastic hydrology, pp. 374-397, Londres, Inglaterra, McGraw-Hill Book Co., SI metric edition, 1988, 492 p.

Machiwal D. y Jha M.K. Comparative evaluation of statistical tests for time series analysis: application to hydrological time series. Hydrological Sciences Journal, volumen 53 (número 2), 2008: 353-366.

Machiwal D. y Jha M.K. Hydrologic time series analysis: theory and practice, capítulo 4: Methods for time series analysis, pp. 51-84, Dordrecht, The Netherlands, Springer, 2012, 303 p.

Madsen H., Lawrence D., Lang M., Martinkova M., Kjeldsen T.R. A review of applied methods in Europe for flood-frequency analysis in a changing environment. European Cooperation in Science and Technology and Centre for Ecology and Hydrology, England, Wallinford, 2013, 180 p.

Manly B.F.J. Statistics for environmental science and management, capítulo 8: Time series analysis, pp. 192-222, Florida, Chapman \& Hall/CRC, Boca Raton, 2001, 326 p.

Martínez-Austria P.F. y Aguilar-Chávez A. Efectos del cambio climático en los recursos hídricos de México. Volumen II, México, SEMARNAT-IMTA, 2008, 118 p.

Martínez-Austria P.F. y Patiño-Gómez C. Efectos del cambio climático en los recursos hídricos de México. Volumen III. Atlas de Vulnerabilidad Hídrica en México ante el Cambio Climático, México, SEMARNAT-IMTA, 2010, 164 p.

Mather J.R. Workbook in Applied Climatology, Exercise II: Skewness, homogeneity and correlation, pp. 9-14, Publications in Climatology, volumen XXX (número 1). Laboratory of Climatology, Nueva Jersey, 1977, 107 p.
Ostle B. y Mensing R.W. Statistics in research, capítulo 7: Regression analysis, pp. 165-236, $3^{\text {a }}$ ed., Iowa, USA, Iowa State University Press, 1975, 596 p.

Raynal-Villaseñor J.A. Cambio climático global: una realidad inequívoca. Ingeniería. Investigación y Tecnología, volumen XII (número 4), 2011: 421-427.

Ruiz-Maya L. Métodos estadísticos de investigación, capítulo 9: condiciones paramétricas del análisis de varianza, pp. 233-249, España, Instituto Nacional de Estadística, 1977, 367 p.

Secretaría de Agricultura y Recursos Hidráulicos (SARH). Boletín Climatológico Núm. 2. Región Hidrológica Núm. 12B (Río Santiago, Poncitlán-Desembocadura). Subsecretaría de Planeación, México, Tomo I, 1977.

Secretaría de Agricultura y Recursos Hidráulicos (SARH). Boletín Climatológico Núm. 3. Región Hidrológica Núm. 37 (El Salado), Subsecretaría de Planeación, México, 1980a.

Secretaría de Agricultura y Recursos Hidráulicos (SARH). Boletín Climatológico Núm. 4. Región Hidrológica Núm. 36 (NazasAguanaval), Subsecretaría de Planeación, México, 1980b.

Shapiro S.S. y Wilk M.B. An analysis of variance test for normality (complete samples). Biometrika, volumen 52, 1965: 591-611.

Shapiro S.S. Selection, fitting and testing statistical models, capítulo 6, pp. 6.1-6.35, en: Handbook of Statistical Methods for Engineers and Scientists, editor Wadsworth H.M., 2a ed., Nueva York, McGraw-Hill Inc, 1998.

Thom H.C.S. Some methods of climatological analysis, technical note Núm. 81, World Meteorological Organization, Genova, Suiza, 1971, 53 p.

World Meteorological Organization (WMO). Climatic change, anexo III: Standard tests of significance to be recommended in routine analysis of climatic fluctuations, pp. 58-71, nota técnica Núm. 79, WMO-Núm. 195, Secretaría de WMO, Genova, Suiza, 1971,79 p. 


\author{
Este artículo se cita: \\ Citación estilo Chicago \\ Campos-Aranda, Daniel Francisco. Búsqueda de tendencias en la \\ precipitación anual del estado de Zacatecas, México; en 30 regis- \\ tros con más de 50 años. Ingeniería Investigación y Tecnología, XVI, \\ 03 (2015): 355-368.

\section{Citación estilo ISO 690} \\ Campos-Aranda D.F. Búsqueda de tendencias en la precipitación \\ anual del estado de Zacatecas, México; en 30 registros con más de \\ 50 años. Ingeniería Investigación y Tecnología, volumen XVI (nú- \\ mero 3), julio-septiembre 2015: 355-368.
}

\section{Semblanza del autor}

Daniel Francisco Campos-Aranda. Obtuvo el título de ingeniero Civil en diciembre de 1972, en la entonces Escuela de Ingeniería de la UASLP. Durante el primer semestre de 1977, realizó en Madrid, España un diplomado en hidrología general y aplicada. Posteriormente, durante 1980-1981 llevó a cabo estudios de maestría en ingeniería en la especialidad de Hidráulica, en la División de Estudios de Posgrado de la Facultad de Ingeniería de la UNAM. En esta misma institución, inició (1984) y concluyó (1987) el doctorado en ingeniería con especialidad en aprovechamientos hidráulicos. Ha publicado artículos principalmente en revistas mexicanas de excelencia: 46 en Tecnología y Ciencias del Agua (antes Ingeniería Hidráulica en México), 18 en Agrociencia y 16 en Ingeniería. Investigación y Tecnología. Es profesor jubilado de la UASLP, desde el $1^{\circ}$ de febrero de 2003. En noviembre de 1989 obtuvo la medalla Gabino Barreda de la UNAM y en 2008 le fue otorgado el Premio Nacional "Francisco Torres H." de la AMH. A partir de septiembre de 2013 vuelve a ser investigador nacional nivel I. 